\title{
Bounds on the dimension of the Brill-Noether schemes of rank two bundles
}

\author{
Ali Bajravani ${ }^{1}$ ii
}

Received: 31 January 2019 / Accepted: 7 June 2019 / Published online: 13 June 2019

(c) Fondazione Annali di Matematica Pura ed Applicata and Springer-Verlag GmbH Germany, part of Springer Nature 2019

\begin{abstract}
The aim of this note is to find upper bounds on the dimension of Brill-Noether locus inside the moduli space of rank two vector bundles on a smooth algebraic curve. We deduce some consequences of these bounds.
\end{abstract}

Keywords Brill-Noether theory · Petri map · Vector bundles

Mathematics Subject Classification $14 \mathrm{H} 51 \cdot 14 \mathrm{H} 60$

\section{Introduction}

Let $C$ be a projective smooth algebraic curve of genus $g$. For nonnegative integers $n$ and $d$, we denote by $U(n, d)$ the moduli space of stable vector bundles of rank $n$ and degree $d$, which is an irreducible scheme of dimension $n^{2}(g-1)+1$. For an integer $k$ with $1 \leq k \leq n+\frac{d}{2}$, the subset

$$
B_{n, d}^{k}=\left\{E \in U(n, d) \mid h^{0}(E) \geq k\right\}
$$

of $U(n, d)$ inherits the structure of a closed sub-scheme of $U(n, d)$. With these notations, $B_{1, d}^{k}$ is the scheme of line bundles of degree $d$ with the space of global sections of dimension at least $k$, which is denoted commonly in the literature by $W_{d}^{k-1}$. In the case of its nonemptiness, $B_{n, d}^{k}$ is expected to be of dimension $n^{2}(g-1)+1-k(k-d+n(g-1))$. As well, for a fixed line bundle $\mathcal{L}$ of degree $d$, we denote the sub-scheme of $U(n, d)$ parameterizing stable bundles $E \in B_{n, d}^{k}$ with determinant $\mathcal{L}$, by $B_{n, \mathcal{L}}^{k}$.

The schemes $B_{n, d}^{k}$, being as natural generalization of the Brill-Noether spaces of line bundles, as well as the spaces $B_{n, \mathcal{L}}^{k}$, have received wide attention from various authors. However, in contrast with extensive results concerning these schemes, specifically the results

Ali Bajravani

bajravani@azaruniv.ac.ir

1 Department of Mathematics, Faculty of Basic Sciences, Azarbaijan Shahid Madani University,

P. O. Box 53751-71379, Tabriz, Islamic Republic of Iran 
on the non-emptiness and existence of components with minimum dimension, there are not, to our knowledge, systematic studies about upper bounds for their dimensions, when $n \geq 2$.

We study this problem for Brill-Noether schemes of rank two bundles, and we obtain upper bounds for $\operatorname{dim} B_{2, d}^{k}$ and $\operatorname{dim} B_{2, K}^{k}$, where $K$ denotes the canonical line bundle on $C$.

The significant point in the rank two case is that a general element in a component of some $B_{2, d}^{k}$, which violates the upper bound and under some specified circumstances, might be assumed to be globally generated. Under the globally generated assumption, a result of Michael Atiyah is applicable. Based on the mentioned result, a globally generated vector bundle can be represented as an extension of a line bundle by the trivial line bundle. Then, using the structure of tangent spaces of $B_{2, d}^{k}$, we relate the kernels of the Petri maps of appropriate bundles in suitable exact sequences. See Theorem 1. As a by-product, we obtain a Mumford type classification result. See Corollary 1.

As for the schemes $B_{2, K}^{k}$, we use an unpublished result of B. Feinberg, which might be considered as a refined version of Atiyah's result. See proposition 1 and lemma 1.

By proving that for an arbitrary smooth curve $C$, a specific component $X \subset B_{2, d}^{2}$ with prescribed circumstances, would be generically smooth of expected dimension; our results push the results of Teixidor [10] and Flamini et al. [5], one step further. See Remark 1(c).

Similar problems, as the problems studied in this paper, have been studied for schemes of Secant Loci in [2-4] by the author.

\section{Preliminaries}

For $E \in B_{n, d}^{k} \backslash B_{n, d}^{k+1}$, the Petri map associated with $E$ controls the tangent vectors of $B_{n, d}^{k}$ at $E$. Indeed, the orthogonal of the image of the Petri map

$$
\mu_{E}^{2}: H^{0}(E) \otimes H^{0}\left(K \otimes E^{*}\right) \rightarrow H^{0}\left(K \otimes E \otimes E^{*}\right),
$$

identifies the tangent space of $B_{n, d}^{k}$ at $E$. Similarly, the tangent space for $B_{2, K}^{k}$ is parameterized by the orthogonal of the image of the symmetric Petri map

$$
\mu_{s, E}^{0}: S^{2} H^{0}(E) \rightarrow H^{0}\left(S^{2} E\right) .
$$

See for example [7].

Assume that $E \in U(2, d)$ and

$$
0 \longrightarrow G \longrightarrow E \longrightarrow L \rightarrow 0,
$$

is an exact sequence of bundles, with $G, L \in \operatorname{Pic}(C)$. Then, there exists a chain of bundles $S^{2}(E) \supset E^{1} \supset E^{2} \supset 0$, such that

$$
\frac{S^{2}(E)}{E^{1}}=2 L, \quad \frac{E^{1}}{E^{2}}=G \otimes L, \quad E^{2}=2 G .
$$

See [8, p. 127]. So, one has two exact sequences

$$
\begin{aligned}
& 0 \longrightarrow 2 G \longrightarrow S^{2} E \longrightarrow \frac{S^{2} E}{2 G} \rightarrow 0, \\
& 0 \longrightarrow G \otimes L \longrightarrow \frac{S^{2} E}{2 G} \longrightarrow 2 L \rightarrow 0 .
\end{aligned}
$$

An unpublished result of B. Feinberg, Lemma 1, is the key tool in the proof of Theorem 4. The lemma is a direct consequence of a characterization result, attributed to B. Feinberg. We quote Teixidor's statement, [11, Lemma 1.1], of this characterizing result in Proposition 1. The proof we present for proposition 1, is quoted from Feinberg's unpublished work in [6]. 
Proposition 1 Denote by $F$ the greatest common divisor of the zeroes of the sections of $E$. Then, either there is a section of $E(-F)$ without zeroes or all sections of $E$ are sections of a line sub-bundle of $E$.

Proof The assertion is an immediate consequence of the following,

Claim 1 Assume that $s_{1}, \ldots s_{r+1}$ are base point free linearly independent sections of $E$ such that the space $\left\langle s_{1}, \ldots, s_{r+1}\right\rangle$ does not contain a nowhere vanishing section. Then, there exists a line sub-bundle $L$ of $E$ such that $\left\langle s_{1}, \ldots, s_{r+1}\right\rangle$ is contained in $H^{0}(C, L)$.

Proof of Claim 1 Set $V:=\left\langle s_{1}, \ldots, s_{r+1}\right\rangle$ and consider the evaluation map

$$
e_{V}: C \times V \rightarrow E, \quad e_{V}(p, s):=s(p) .
$$

We show that $\operatorname{ker}\left(e_{V}\right)$ is a vector bundle of rank $r$ and consequently the saturation of the image of $e_{V}$ is a line bundle. Observe that the hypothesis of being base point free is equivalent to the fact that the dimension of $\operatorname{ker}\left(e_{V}\right)_{p}$ is at most $r$ for all $p$ in $C$. If, on the other hand, the rank of $\operatorname{ker}\left(e_{V}\right)$ is generically less than $r$, then the dimension of the image of $\operatorname{ker}\left(e_{V}\right)$ under the composition:

$$
\operatorname{ker}\left(e_{V}\right) \rightarrow C \times V \rightarrow V
$$

is at most $r$. This, however, would imply that $V$ has a nowhere vanishing section, which is a contradiction. Therefore, $\operatorname{ker}\left(e_{V}\right)$ is a vector bundle of rank $r$ and $e_{V}$ surjectively maps onto a line sub-bundle in $E$. This completes the proof of Claim 1.

Lemma 1 Any vector bundle $E$ with $h^{0}(E)=k \geq 2$ admits an extension as

$$
0 \longrightarrow \mathcal{O}(D) \stackrel{i}{\longrightarrow} E \stackrel{\pi}{\longrightarrow} L \rightarrow 0
$$

where $D$ is an effective divisor and either $h^{0}(\mathcal{O}(D))=1$ or $h^{0}(\mathcal{O}(D))=k$.

Motivated by Lemma 1, two types of bundles with sections are distinguishable.

Definition 1 A vector bundle $E$ with $h^{0}(E)=k \geq 1$ will be said of first type if it admits an extension as (8) with $h^{0}(\mathcal{O}(D))=k$. Otherwise, we call $E$ of second type.

\section{Main results}

Theorem 1 Let $k, d$ be integers with $3 \leq d \leq 2 g-2,2 \leq k \leq 2+\frac{d}{2}$. Then,

$$
\operatorname{dim} B_{2, d}^{k} \leq 2(g-1)+d-2 k+1 .
$$

Proof Observe first that if a general element $E$ of an irreducible component $X$ of $B_{2, d}^{k}$ satisfies $h^{0}(E) \geq k+1$, then we can consider $X$ as a component of $B_{2, d}^{k+1}$. Therefore for general $E \in X$ one may assume $h^{0}(E)=k$. Assume that $d$ is a minimum integer such that for some suitable $k$ there exists a component $X$ of $B_{2, d}^{k}$ with $\operatorname{dim} X \geq 2(g-1)+d-2 k+2$. Then, a general element $E$ in $X$ is globally generated. Indeed otherwise we obtain $\operatorname{dim} B_{2, d-2}^{k-1} \geq 2(g-1)+d-2 k+2$, which is impossible by minimality of $d$. Therefore, by [1, Theorem 2], a general element $E$ in $X$ has a trivial line bundle as its line sub-bundle. Furthermore $E$ admits a representation as

$$
0 \longrightarrow \mathcal{O}_{C} \stackrel{i}{\longrightarrow} E \stackrel{\pi}{\longrightarrow} L \longrightarrow 0
$$


with the property that the sections of $L$ belonging to the image of $H^{0}(\pi)$ have at most one number of base points. Indeed, if $L$ has the points $p, q$ as its base points, then $h^{0}(E(-p-$ $q)) \geq k-2$. This implies that

$$
\operatorname{dim} B_{2, d-4}^{k-2} \geq 2(g-1)+d-2 k+2,
$$

which is absurd again by minimality of $d$. Take an extension as (10) and consider the exact sequence

$$
0 \longrightarrow H^{0}\left(\mathcal{O}_{C}\right) \stackrel{H^{0}(i)}{\longrightarrow} H^{0}(E) \stackrel{H^{0}(\pi)}{\longrightarrow} V \longrightarrow 0
$$

where $V$ is the image of the map $H^{0}(\pi): H^{0}(E) \longrightarrow H^{0}(L)$. The exact sequence (11) together with various Petri maps gives rise to a commutative diagram as

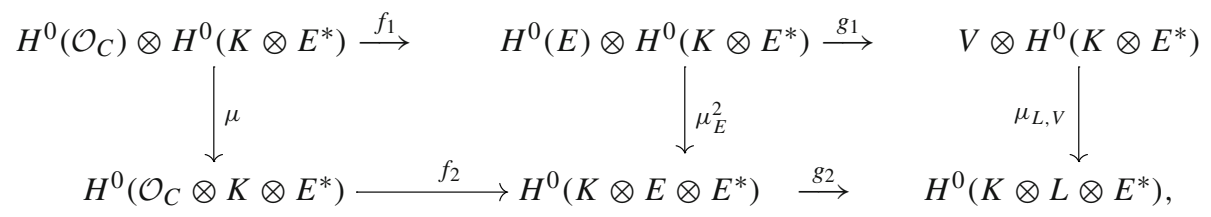

in which the maps $f_{1}$ and $f_{2}$ are injective and $g_{1}$ is surjective. Observe furthermore that the map $\mu$ is an isomorphism. The snake lemma applied to this situation implies that

$$
\operatorname{dim} \operatorname{ker} \mu_{E}^{2}=\operatorname{dim} \operatorname{ker} \mu_{L, V} \text {. }
$$

According to the assumption concerning dimension of $X$, we obtain

$$
\operatorname{dim} \operatorname{ker} \mu_{L, V} \geq(k-1)(2 g-2-d+k-1) .
$$

Assuming $V=\left\langle v_{1}, \ldots, v_{k-1}\right\rangle$ and setting

$$
V_{i}:=\left\langle v_{1}, \ldots, v_{i}\right\rangle, \quad i=2, \ldots, k-1,
$$

we would have $\operatorname{dim} \operatorname{ker} \mu_{L, V_{i}}-\operatorname{dim} \operatorname{ker} \mu_{L, V_{i-1}} \leq h^{0}\left(K \otimes E^{*}\right)$. These together with the base-point-free pencil trick applied to the map

$$
\mu_{L, V_{2}}: V_{2} \otimes H^{0}\left(K \otimes E^{*}\right) \rightarrow H^{0}\left(K \otimes L \otimes E^{*}\right),
$$

implies $h^{0}\left(K \otimes E^{*} \otimes L^{*}(B)\right) \geq 2(2 g-2-d+k)-(k-1)$, where $B$ is the base locus of the sections of $V_{2}$. Note also that $0 \leq \operatorname{deg}(B) \leq 1$. Therefore,

$$
h^{0}\left(K \otimes E^{*} \otimes L^{*}\right) \geq 2(2 g-2-d+k)-k .
$$

If $\frac{4 g-3}{3} \leq d$ then, as $\operatorname{deg}\left(K \otimes E^{*} \otimes L^{*}\right)<0$ and $K \otimes E^{*} \otimes L^{*}$ is stable, one has $h^{0}\left(K \otimes E^{*} \otimes L^{*}\right)=0$, which is in contradiction with inequality (14).

Recall that $h^{0}(E \otimes L)=h^{0}\left(K \otimes E^{*} \otimes L^{*}\right)+3 d-2(g-1) \geq 2(g-1)+d+k$. Now if $d \leq \frac{4 g-4}{3}$, then $\mu(E \otimes L) \leq 2 g-3$. Observe furthermore that $E \otimes L$ is stable. As a consequence of Propositions 3 and 4 of [9], the Clifford theorem for vector bundles for such a this situation asserts that $h^{0}(E \otimes L) \leq \frac{\operatorname{deg}(E \otimes L)+\mathrm{rk}(E \otimes L)}{2}$, by which we obtain $2(g-1)+d+k \leq 1+\frac{3 d}{2}$. Consequently, we get $d+k \leq 0$, which is absurd.

Theorem 2 If $g \geq 5$, then

$$
\operatorname{dim} B_{n, d}^{2} \leq n(n-1)(g-1)+d-3 .
$$


Proof Assume that $X$ is an irreducible component of $B_{n, d}^{2}$ and $E$ is a general element of $X$. Assume moreover, as in theorem 1, that a general element $E \in X$ satisfies $h^{0}(E)=2$. Observe that, using a diagram as in diagram (12), we can obtain an equality as (13), by which, if $E$ turns out to be of second type, then $\mu_{E}^{2}$ would be injective. So $X$ has to be generically smooth, and it has to have the expected dimension, which is certainly smaller than the claimed bound.

If a general element of $X$ turns to be of first type, then

$$
\operatorname{dim} X \leq n(n-1)(g-1)+d-4 .
$$

Indeed, if a general element $E \in X$ admits a presentation as

$$
0 \rightarrow H \rightarrow E \rightarrow F \rightarrow 0,
$$

where $H$ is a line bundle with $h^{0}(H)=2, \operatorname{deg}(H)=d_{1}$ and $\operatorname{rk}(F)=n-1$, then since the stable bundles deform to non-stable ones, we can assume in counting that $F$ is stable as well. So the dimension of the set of bundles as $F$, is bounded by $\operatorname{dim} U\left(n-1, d-d_{1}\right)=$ $(n-1)^{2}(g-1)+1$. Meanwhile, the line bundles as $H$ would vary in a subset $\mathcal{H}$ of $B_{1, d_{1}}^{2}$ and the Martens' theorem asserts that $\operatorname{dim} \mathcal{H} \leq d_{1}-2\left(\operatorname{dim} \mathcal{H}\right.$ can be $d_{1}-2$ if $C$ is hyper-elliptic and $\operatorname{dim} \mathcal{H} \leq d_{1}-3$ otherwise). Therefore, the dimension of $X$ would be bounded by

$$
\left[d_{1}-2\right]+\left[(n-1)^{2}(g-1)+1\right]+\left(h^{1}\left(H \otimes F^{*}\right)-1\right) .
$$

Observe that $h^{1}\left(H \otimes F^{*}\right)=(n-1)(g-1)+d-n d_{1}$ by Riemann-Roch. Moreover, $d_{1} \geq 2$ and so

$$
\operatorname{dim} X \leq n(n-1)(g-1)+d-2-d_{1}(n-1) \leq n(n-1)(g-1)+d-4,
$$

as required.

Motivated by [5, Theorem 1.2], one can sharpen the bound in Theorem 1 under some restrictions on the numbers $r, d$, as

Theorem 3 Let $k, d$ be integers with $3 \leq d \leq 2 g-2-\frac{k}{2}, 2 \leq k \leq 2+\frac{d}{2}$. Then, if $k \geq 3$, then $\operatorname{dim} B_{2, d}^{k} \leq 2 g+d-4 k$. While for $k=2$, the integer $d$ can vary in the set $\{3, \ldots, 2 g-5\}$ with the same bound for $\operatorname{dim} B_{2, d}^{2}$.

Proof The argument of proof of Theorem 1 goes through to deduce the result. Notice that the further restriction on $d$ in the case $k=2$ was needed to be imposed, because the quantity $2 g+d-4 k$ turns out to be smaller than the expected dimension for $2 g-4 \leq d \leq 2 g-2$.

\subsection{The case of canonical determinant}

Theorem 4 For an integer $k$ with $2 \leq k \leq g+1$, any irreducible component $X$ of $B_{2, K}^{k}$ satisfies

$$
\operatorname{dim} X \leq 3 g-2 k-2
$$

Proof Let $X$ be an irreducible component of $B_{2, K}^{k}$ and a general element $E$ of $X$ satisfies $h^{0}(E)=k$. Assume that a general member $E \in X$ is of second type and set $\gamma:=\operatorname{dim} X$. Then, one has

$$
3 g-3-\left(\operatorname{dimim} \mu_{s, E}^{0}\right) \geq \gamma,
$$


where $\mu_{s, E}^{0}$ is the symmetric Petri map associated with $E$ as in (2). So

$$
\operatorname{dim} \operatorname{ker} \mu_{s, E}^{0} \geq \gamma+\frac{k(k+1)}{2}+3-3 g .
$$

The exact sequence $0 \rightarrow \mathcal{O}(2 D) \rightarrow S^{2} E \rightarrow \frac{S^{2} E}{\mathcal{O}(2 D)} \rightarrow 0$, arising from the exact sequence (5), gives rise to a commutative diagram as

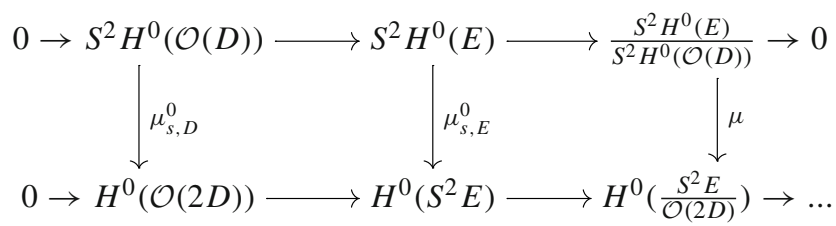

Since $S^{2} H^{0}(\mathcal{O}(D))=\mathbb{C}$, the map $\mu_{s, D}^{0}$ turns to be injective. This together with the snake lemma gives an inequality as

$$
\operatorname{dim} \operatorname{ker} \mu \geq \operatorname{dim} \operatorname{ker} \mu_{s, E}^{0} .
$$

Therefore, using inequality (18), we obtain

$$
\operatorname{dim} \operatorname{ker} \mu \geq \gamma+\frac{k(k+1)}{2}+3-3 g .
$$

Let $V$ be as in the proof of Theorem 1 and observe by effectiveness of $D$ that the vector space $V$ can be considered as a subspace of $H^{0}(\mathcal{O}(D) \otimes L)$. Similar to the previous argument, the exact sequence

$$
0 \rightarrow \mathcal{O}(D) \otimes L \rightarrow \frac{S^{2} E}{\mathcal{O}(2 D)} \rightarrow 2 L \rightarrow 0,
$$

as well arising from the exact sequence (6), together with the equality

$$
S^{2} H^{0}(E)=S^{2} V \oplus V \oplus \mathbb{C},
$$

leads to the following commutative diagram of bundles

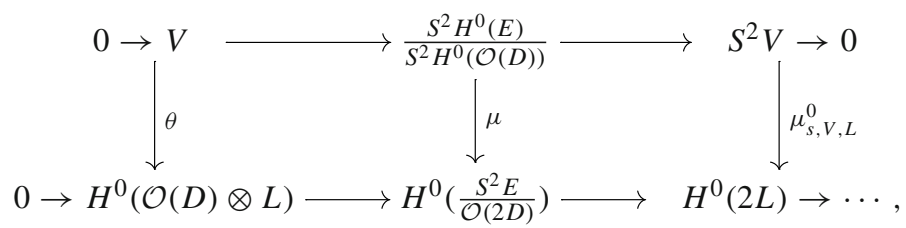

where $\mu_{s, V, L}^{0}$ is the symmetric Petri map of $L$ restricted to $S^{2} V$ and $\theta$ is the inclusion map. Once again, as a consequence of the injectivity of $\theta$ and the snake lemma, we obtain

$$
\operatorname{dim} \operatorname{ker} \mu_{s, V, L}^{0} \geq \operatorname{dim} \operatorname{ker} \mu,
$$

by which together with (21) an inequality as

$$
\operatorname{dim} \operatorname{ker} \mu_{s, V, L}^{0} \geq \gamma+\frac{k(k+1)}{2}+3-3 g
$$


would be obtained. This, in combination with $\operatorname{dim} \operatorname{ker} \mu_{s, V, L}^{0} \leq \operatorname{dim} S^{2} V-\operatorname{dim} V$, implies

$$
\gamma \leq 3 g-2 k-2
$$

as required.

Finally, if $\operatorname{dim} X \geq 3 g-2 k-1$, then a general member $E$ of $X$ fails to be of first type. Indeed otherwise, assume that a general member $E \in X$ admits a presentation as

$$
0 \longrightarrow \mathcal{O}(D) \stackrel{i}{\longrightarrow} E \stackrel{\pi}{\longrightarrow} K \otimes \mathcal{O}(-D) \rightarrow 0,
$$

with $\operatorname{deg}(D)=t$. Then, the stability of $E$ implies that $t \leq g-2$, and we would have

$$
\operatorname{dim} B_{1, t}^{k}+h^{1}\left(\mathcal{O}(D) \otimes L^{-1}\right)-1 \geq 3 g-2 k-1 .
$$

This, since $h^{0}\left(\mathcal{O}(D) \otimes L^{-1}\right)=0$ by stability of $E$, implies that

$$
\operatorname{dim} B_{1, t}^{k} \geq 2 t-2 k+3
$$

which is absurd by Martens' theorem.

\section{Remarks and Corollaries}

Corollary 1 (Mumford's Theorem for rank two bundles) If $C$ is non-hyper-elliptic of genus $g \geq 19$ and if for some $k, d$ with $0<2 k-2 \leq d \leq 2 g-\frac{3}{2} k-\frac{7}{2}$ one had $\operatorname{dim} B_{2, d}^{k}=$ $2 g+d-4 k$, then either $C$ is trigonal, or bi-elliptic, or a smooth plane quintic.

Proof Assume that $X$ is an irreducible component of $B_{2, d}^{k}$ with $\operatorname{dim} X=2 g+d-4 k$. If a general element $E \in X$ is of first type and has $k$ number of independent sections, then one has $\operatorname{dim} B_{1, t}^{k} \geq 2(t-2 k+1)$ for some integer $t$ with $0<2(k-1) \leq t \leq g-2$. This, by Mumford's theorem, might occur only if $t-2 k+1=0$ by which the equality $\operatorname{dim} B_{1,2 k-1}^{k}=0$ holds. So $\mathcal{O}(D) \in B_{1,2 k-1}^{k}$, which may happen only in the case that either $C$ is trigonal, or bi-elliptic, or a smooth plane quintic.

Claim 2 If $E$ fails to be of first type, then for general points $p_{1}, \ldots p_{\left[\frac{k-2}{2}\right]} \in C$, the stable vector bundle $E\left(-p_{1}-\ldots-p_{\left[\frac{k-2}{2}\right]}\right)$ would fail to admit an extension of first type.

Proof of Claim 2 Assume first that $k$ is even. If the stable vector bundle $E\left(-p_{1}-\ldots-p_{\left[\frac{k-2}{2}\right]}\right)$ turns to be of first type, then there exists a set of line bundles $H$ with $h^{0}(H) \geq 2$ and $\operatorname{deg} H \leq g-2$. Tensoring $H$ with $\mathcal{O}\left(p_{1}+\cdots+p_{t}\right)$ for general points $p_{1}+\cdots+p_{t}$, if necessary, we can assume that $H \in B_{1, g-2}^{2}$. Therefore we obtain $\operatorname{dim} B_{1, g-2}^{2} \geq 2 g+d-4 k+\left[\frac{k-2}{2}\right]$. This by Martens' theorem implies that $7 k-8 \geq 2 g+2 d$. On the other hand, the inequalities $2 k-2 \leq d$ and $2 k-2 \leq 2 g-\frac{3}{2} k-\frac{7}{2}$ imply $4 k \leq 2 d+4$ and $3 k \leq \frac{12}{7} g-\frac{9}{7}$, respectively. Summing up all the inequalities we obtain $g \leq 18$, which is absurd. If $k$ is an odd number, then the argument goes verbatim to prove the claim by replacing $B_{1, g-2}^{3}$ with $B_{1, g-2}^{2}$. So the Claim 2 is established.

If a general bundle $E \in X$ turns to be of second type and if $k=2 n$, then the scheme $B_{2, d-2\left[\frac{k-2}{2}\right]}^{2}$ contains a subset $Y$ which is at least of dimension $2 g+d-4 k+\left[\frac{k-2}{2}\right]$ and its general member is a vector bundle of second type. According to the work of M. Teixidor in [10], such a subset $Y$, if non-empty, is of expected dimension and the expected dimension is strictly smaller than $2 g+d-4 k+\left[\frac{k-1}{2}\right]$ for $d$ in the given range. This is a contradiction. 
If $k=2 n+1$, with similar assumption on $E$, the scheme $B_{2, d-2\left[\frac{k-2}{2}\right]}^{3}$ would contain a subset $Y$ which is at least of dimension $2 g+d-4 k+\left[\frac{k-2}{2}\right]$ and its general member is a vector bundle of second type. This possibility can be excluded by another work of M. Teixidor in [11].

Corollary 2 The scheme $B_{2, K}^{2}$ is reduced and irreducible of dimension $3 g-6$.

Proof The upper bound $3 g-6$ on the dimension is obvious by theorem 4 . If $E \in B_{2, K}^{2} \backslash B_{2, K}^{3}$, then the petri map $\mu_{2, K, E}^{0}$ turns to be injective. Indeed, if $E$ is a bundle of first type, then using diagram (19), since $\frac{S^{2} H^{0}(E)}{S^{2} H^{0}(\mathcal{O}(D))}$ vanishes, the Petri map $\mu_{s, E}^{0}$ would be injective. While if $E$ is of second type, since $S^{2} V$ is one dimensional, then $\mu_{s, V, L}^{0}$ is injective and so the map $\mu$ is injective by (23). This together with (20) implies that the Petri map $\mu_{s, E}^{0}$ is again injective. So we obtain

$$
\text { Sing } B_{2, K}^{2}=B_{2, K}^{3} \text {. }
$$

Since $B_{2, K}^{2}$ is of expected dimension, so it might be reducible only if its singular locus is, by [12], of codimension $\leq 1$; i.e., $\operatorname{dim} B_{2, K}^{3} \geq 3 g-7$, by (27). This is a contradiction, because by Theorem (4) the locus $B_{2, K}^{3}$ is of dimension at most $3 g-8$.

Since, again by theorem 4 , no irreducible component of $B_{2, K}^{2}$ is contained entirely in $B_{2, K}^{3}$, so $B_{2, K}^{2}$ would be reduced.

Using Lemma 2, the bound in theorem 4 can be sharpened for odd values of $k$.

Lemma 2 If $\mathcal{L}$ is a globally generated line bundle on $C$ with $h^{0}(\mathcal{L})=s+1$, then the set of vector bundles of second type $E \in B_{2, d, \mathcal{L}}^{k}(k=1,2)$, if non-empty, is of dimension at most $s+d-4$, (res. at most of dimension $\left.\frac{d}{2}+2(s-3)\right)$, if $k=2$ (res. if $k=3$ ).

Proof For $k=2$, with notations as in proof of [10, Page 124], the dimension of the set of vector bundles $E \in B_{2, d, \mathcal{L}}^{k}$ of second type is bounded by

$$
\operatorname{dim}\{D\}+h^{0}(\mathcal{L}(-D))-1+\operatorname{dim}\langle\dot{D}\rangle-\left(h^{0}(E)-1\right)
$$

where $D$ is a divisor in the linear series $|\mathcal{L}(-D)|$ and $t=\operatorname{deg}(D)$. It is now an easy argument to see that this quantity is bounded by

$$
t+(s-1)+(d-t-2)-1=d+s-4 .
$$

If $k=3$, then a close analysis in the proof of [11, Theorem 2], implies that the dimension of the bundles $E \in \operatorname{dim} B_{2, d, \mathcal{L}}^{3}$ which are of second type, is bounded by the quantity $\operatorname{dim}\{D\}+$ $\operatorname{dim} \operatorname{Grass}(2, \mathcal{L}(-D))+\operatorname{dim}\left\langle D_{1} \cap \dot{D}_{2}\right\rangle-\left(h^{0}(E)-1\right) \leq \frac{d}{2}+2(s-3)$, as required.

Corollary 3 If $k$ is odd, then

$$
\operatorname{dim} B_{2, K}^{k} \leq 3 g-2 k-3
$$

Proof An irreducible component $X$ of $B_{2, K}^{k}$ whose general member is a bundle of first type has dimension $\leq 3 g-2 k-3$, because otherwise one obtains $\operatorname{dim} B_{1, t}^{k} \geq 2 t-2 k+2$ for some $k$ and $t$ with $0<2 k-2 \leq t \leq g-2$. This is obviously absurd.

Assume that $\operatorname{dim} X=3 g-2 k-2$ and set $k-1=2 n$. 
Claim 3 If a general $E \in X$ fails to be of first type, then for general points $p_{1}, \ldots p_{i} \in C$ with $1 \leq i \leq \frac{k-1}{2}$ the stable vector bundle $E\left(-p_{1}-\ldots-p_{i}\right)$ would fail to admit an extension of first type.

The proof of Claim 3 is similar to the proof of Claim 2 in corollary 1.

Lemma (2) together with Claim 3 implies that if a general element of $X$ fails to be of first type then

$$
3 g-2 k-2 \leq(n-1)+\operatorname{dim} B_{2,2 g-2 n, K\left(-2 p_{1}-\ldots-2 p_{n-1}\right)}^{3} \leq 3 g-4 n-5,
$$

which is absurd.

Remark 1 (a) If $C$ is an arbitrary 3-gonal curve, then Theorem 3 together with Theorem [5, Thm. 1.2(b)] imply dim $B_{2, d}^{2}=2 g+d-8$. Indeed, Theorem [5, Thm. 1.2(b)] establishes this result for a general 3-gonal curve and so for non-general 3-gonal curves, one has $\operatorname{dim} B_{2, d}^{2} \geq 2 g+d-8$. Now, Theorem 3 applied to such a non-generic curve implies the equality for any 3 -gonal curve.

(b) According to Theorem 2, one immediately re-obtains $\operatorname{dim} B_{n, n(g-1)}^{2}=n^{2}(g-1)-3$. Meanwhile, by the same theorem, an immediate prediction suggests the quantity $n(n-$ $1)(g-1)+d-2 k+1$ as a bound to the dimension of $B_{n, d}^{k}$ when $n \geq 3, k \geq 3$. A proof to this expectation is unknown to me. Such a bound re-obtains Marten's bound on the dimension of the Brill-Noether schemes of line bundles.

(c) The proofs of Theorems 1 and 2 indicate that the Petri map is injective at the bundles $E \in B_{n, d}^{2}$ which are of second type. Therefore

$$
\text { Sing } B_{n, d}^{2} \subseteq B_{n, d}^{3} \cup \mathcal{E}_{1},
$$

where $\mathcal{E}_{1}$ denotes the set of bundles $E \in B_{n, d}^{2}$ of first type. This reproves the generic smoothness of the locus' introduced by Teixidor [10] and Flamini et al. [5].

Acknowledgements The author wishes to thank F. Flamini, P. Newstead, and M. Teixidor for their valuable hints and for sharing their knowledge. I specially thank G. H. Hitching whose careful reading and comments changed the previous manuscript of this paper, considerably. Teixidor supported me by sending a draft of the unpublished paper [6] at the right time; to her, I express my double gratitude. I also thank the anonymous referee for his/her very useful comments on the paper.

\section{References}

1. Atiyah, M.: Vector bundles over an elliptic curve. Proc. Lond. Math. Soc. (3) 7, 414-452 (1957)

2. Bajravani, A.: Martens-Mummford theorems for Brill-Noether schemes arising from very ample line bundles. Arc. Math. 105, 229-237 (2015)

3. Bajravani, A.: Remarks on the geometry of secant loci. Arc. Math. 108, 373-381 (2016)

4. Bajravani, A.: A note on the tangent cones of the scheme of Secant Loci. Rendiconti del Circolo Matematico di Palermo Series 2 67, 599-608 (2018)

5. Choi, Y., Flamini, F., Kim, S.: Brill-Noether theory of rank two vector bundles on a general $v$-Gonal curve. Proc. AMS 146, 3233-3248 (2018)

6. Feinberg, B.: On the Dimension and Irreducibility of Brill-Noether Loci. Unpublished paper

7. Grzegorczyk, I., Teixidor, M.: Brill-Noether theory for stable vector bundles. In: Bradlow, S., BrambilaPaz, L., Garcia-Prada, O., Ramanan, S. (eds.) Moduli Spaces of Vector Bundles. LMS Lecture Note Series 359, pp. 29-50. Cambridge University Press (2009)

8. Hartshorne, R.: Algebraic Geometry, Graduate Texts in Mathematics 52. Springer, New York (1977)

9. Re, R.: Multiplication of sections and Clifford bounds for stable vector bundles on curves. Commun. Algebra 26, 1931-1944 (1998)

10. Teixidor, M.: Brill-Noether theory for vector bundles of rank 2. Tohuku Math. J. 43, 123-126 (1991) 
11. Teixidor i Bigas, M.: On the Gieseker-Petri map for rank 2 vector bundles. Manuscr. Math. 75(4), 375-382 (1992)

12. Teixidor, M.: For which Jacobi Varieties is Sing $\Theta$ reducible? J. Reine Angew. Math. 354, 141-149 (1984)

Publisher's Note Springer Nature remains neutral with regard to jurisdictional claims in published maps and institutional affiliations. 